Citar como: Roncero, C., Fuste, G., Barral, C., Rodríguez-Cintas, L., Eiroa-Orosa, F. J., \& Casas, M. (2009). Manejo terapéutico y comorbilidades del paciente con dependencia a opiáceos, en programa de terapia sustitutiva: Estudio PROTEUS. Trastornos Adictivos, 11(4), 266-270. http://doi.org/10.1016/S1575-0973(09)73470-1

\title{
Manejo terapéutico y comorbilidades del paciente con dependencia a opiáceos, en programa de terapia sustitutiva: ESTUDIO PROTEUS
}

Carlos Roncero ${ }^{\mathrm{a}}$; Gideoni Fuste ${ }^{\mathrm{b}}$; Carmen Barral ${ }^{\mathrm{b}}$, Laía Rodríguez-Cintas ${ }^{\mathrm{c}}$, Francisco José Eiroa-Orosa $^{\mathrm{c}}$, Miguel Casas ${ }^{\mathrm{d}}$

a Coordinador del CAS Vall d’Hebron. Servicio de Psiquiatría del Hospital Universitario Vall d’Hebron. Profesor asociado de la Universidad Autónoma de Barcelona

${ }^{\mathrm{b}}$ Psiquiatra. CAS Vall d’Hebron. Servicio de Psiquiatría del Hospital Universitario Vall d'Hebron. Universidad Autónoma de Barcelona

${ }^{c}$ Psicólogo. CAS Vall d’Hebron. Servicio de Psiquiatría del Hospital Universitario Vall d’Hebron

d Jefe de Servicio de Psiquiatría del Hospital Universitario Vall d’Hebron. Catedrático de Psiquiatría. Universidad Autónoma de Barcelona

Correspondencia:

Carlos Roncero Alonso

Hospital Universitario Vall d’Hebron; Servicio de Psiquiatría, Escuela de enfermería $5^{\circ}$ planta; Passeig Vall d’hebron 119-129; código postal 08035; Barcelona.

Correo electrónico croncero@vhebron.net 


\section{RESUMEN}

Objetivo. Analizar la necesidad de realizar un estudio epidemiológico de pacientes dependientes de opiáceos centrado en conocer más sus tratamientos y comorbilidades médicas y psiquiátricas.

Métodos. Se examinó la necesidad de tener datos disponibles en nuestro medio sobre manejo clínico de estos pacientes, el tipo de tratamiento y se las posibles comorbilidades. No existen datos completos, representativos de los pacientes españoles. Por ello se seleccionó una muestra representativa a nivel nacional, a partir de la población de pacientes dependientes de opiáceos en programas de mantenimiento con agonistas opiáceos que acudían a los centros asistenciales de drogodependencias.

Resultados. El estudio final, incluyó una población representativa de 624 pacientes dependientes a opiáceos en programas de mantenimiento con agonistas, procedentes de 74 centros de asistencia al dependiente a opiáceos de toda España.

Conclusiones. Se conocen poco los datos sobre la comorbilidad psicopatológica y médica y los tratamientos farmacológicos en pacientes dependientes de opiáceos. Es necesario realizar un estudio epidemiológico amplio que actualice la realidad de la práctica clínica habitual del paciente dependiente a opiáceos en España (manejo terapéutico, comorbilidades, etc.) valorando la gravedad de su adicción, la repercusión sobre el tipo de tratamiento, la presencia de patología dual y las repercusiones médicas.

Palabras Clave: dependencia de opiáceos, manejo clínico, patología dual, comorbilidad médica, Estudio PROTEUS 


\begin{abstract}
Background: The purpose of this study was to analyze the need for an epidemiological study of opiate-dependent patients aiming to improve the knowledge about their treatment and medical and psychiatric comorbidities.
\end{abstract}

Methods. Data on clinical management, treatment type, and comorbidities in Spain were examined. There are no comprehensive data, representative of the Spanish patients. Therefore, a national representative sample was selected from opiate-dependent patients ongoing replacement therapy programs, attending care centres for opiate-dependent patients.

Results. The representative sample included 624 opiate-dependent patients ongoing opiate replacement therapy programs from 74 Drug-dependent rehabilitation centres in Spain.

Conclusions. Data on therapeutic management and psychiatric and medical comorbidities in opiate-dependent patients are not well known. There is a need for a comprehensive epidemiological study to update the reality of clinical practice of opioiddependent patients in Spain (therapeutic management, comorbidities, etc.) assessing the severity of their addiction, the impact on the type of treatment, the presence of dual pathology and medical implications.

Keywords: opiate dependence, dual diagnosis, clinical management, medical comorbidity, PROTEUS study. 


\section{1-Introducción}

Se calcula que entre 1 y 8 casos por 1000 adultos (entre 15 y 64 años de edad) consumen opiáceos en Europa ${ }^{1}$. En España, según los datos del Plan Nacional sobre Drogas, en el año 2005, el número de usuarios, en los distintos recursos asistenciales, con un diagnóstico de dependencia de opiáceos ${ }^{2}$ fue de 172.980. Alrededor del $48 \%$ pacientes realizaban programas de mantenimiento con metadona, 79.809 (46\%) fueron atendidos en centros ambulatorios de asistencia, 3.750 pacientes fueron ingresados en unidades de desintoxicación hospitalaria y 6.047 fueron atendidos en comunidades terapéuticas.

El tratamiento de una dependencia de opiáceos persigue como primer objetivo la abstinencia completa. En los casos en los que ésta no sea un objetivo realista a corto plazo, se opta por los tratamientos sustitutivos o de mantenimiento. Son tratamientos de probada eficacia en los que se sustituye el opiáceo de consumo por otro, que se administra de forma supervisada y por un tiempo prolongado. El objetivo no es solo evitar síntomas de abstinencia, si no las recaídas en el consumo. En nuestro medio, el opiáceo utilizado en estos programas es comúnmente la metadona ${ }^{2}$.

La administración de este fármaco se incluye dentro de los programas denominados programa de mantenimiento con metadona (PMM). No todos los PMM son iguales. Si bien el denominador común es la utilización de la metadona como fármaco de sustitución opiáceo, el concepto PMM incluye muchos tipos de programas diferentes: desde los que se limitan a la administración de metadona sin ningún otro apoyo adicional, hasta los que ofrecen un abordaje más completo como el tratamiento de la psicopatología comórbida, la evaluación y tratamiento de otras patologías orgánicas como la infección por el virus de la inmunodeficiencia humana (VIH), el virus de la hepatitis B (VHB) y el virus de la hepatitis C (VHC), etc. Asimismo, la propia administración de la metadona puede estar sometida a muchas diferencias en cuanto a dosis, duración y forma de administración, según los distintos programas.

Durante los últimos años ha habido un interés por mejorar el tratamiento de los pacientes dependientes de opiáceos y se ha demandado el poder utilizar otras alternativas de tratamiento ${ }^{3}$. El uso de otros agonistas opiáceos, como la buprenorfina o de la buprenorfina/naloxona es una alternativa eficaz ${ }^{4}$. En España desde el año 2008 comenzó su prescripción y progresivamente se ha convertido en una práctica más 
habitual, ya que posiblemente constituya una opción farmacéutica más segura que la metadona para utilizar en los centros basados en la comunidad ${ }^{5}$.

\section{2-Comorbilidad de los dependientes de opiáceos}

El consumo de heroína se asocia con frecuencia a todo un conjunto de complicaciones médicas ${ }^{6}$ principalmente infecciosas (tuberculosis, hepatitis por VHB y VHC, VIHSIDA, entre las más graves y frecuentes) pero también no infecciosas (cardiovasculares, inmunitarias, gastrointestinales, dermatológicas, etc.). Los cambios en los últimos años en el consumo de heroína, sustituyendo la vía parenteral por la inhalada o fumada o el desarrollo de los programas de asistencia y reducción de daños, habían supuesto un descenso de la prevalencia de las enfermedades infecciosas, Sin embargo, en los últimos años parece que se ha reactivado el uso intravenoso ${ }^{2}$, lo que podría suponer una reaparición de las patologías infecciosas más graves.

En España existen datos muy valiosos sobre los pacientes dependientes de opiáceos, procedentes de los distintos informes del Observatorio Para el Plan Nacional sobre Drogas $^{2}$. Sin embargo, aspectos como la presencia de patología dual, comorbilidades médicas, tratamientos concomitantes recibidos por los pacientes, no han sido totalmente dilucidados. Existen descripciones clínicas ${ }^{7}$, estudios de cohortes específicas ${ }^{8}$, trabajos epidemiológicos, habitualmente locales, que han revelado un alto grado de comorbilidad con otros trastornos psiquiátricos en sujetos con dependencia de opiáceos ${ }^{9-11}$. Algunos trabajos han sido realizados con una metodología muy rigurosa de investigación y han supuesto importantes avances a nivel internacional, en el conocimiento de la comorbilidad $^{12}$.

Se conoce que la prevalencia de trastornos psiquiátricos en dependientes de opiáceos se sitúa entre un 44-93\%, siendo el trastorno depresivo el trastorno mental más frecuente $(\text { entre } 4-54 \%)^{8-12}$. Esta gran variabilidad entre los diferentes estudios puede reflejar diferencias entre las poblaciones estudiadas (sesgos locales, muestras con ambos sexos en relación con muestras solamente de hombres, tipo de tratamiento recibido por los pacientes, agonista opiáceo utilizado, instrumentos psicométricos empleados....etc. $)^{7,9}$. Todo ello permite afirmar que muchos aspectos relacionados con la comorbilidad y el manejo del tratamiento permanecen aún desconocidos, en la población general de pacientes en tratamiento con agonistas opiáceos. 


\section{3-Métodos}

En el contexto actual parece necesario realizar un estudio epidemiológico amplio, con representación geográfica adecuada, que actualice la realidad de la práctica clínica habitual del paciente dependiente a opiáceos en España (manejo terapéutico, comorbilidades, etc.) valorando la gravedad de su adicción y sus repercusiones médicas y en el tratamiento.

Para ello, se diseño el estudio PROTEUS. Se seleccionó una población representativa de pacientes dependientes a opiáceos de entre todos aquellos procedentes de los centros de asistencia al dependiente a opiáceos (centros ambulatorios de asistencia y centros que realizan programas de mantenimiento con metadona, principalmente) de todo el territorio nacional.

Para cumplir con el objetivo del estudio, se planificó incluir en el estudio un total de 800 pacientes con dependencia a opiáceos. Este número de pacientes representa alrededor del $1 \%$ del total de pacientes dependientes a opiáceos en España. Para tener una muestra representativa de la población de pacientes con dependencia a opiáceos de toda España, la selección de los pacientes se realizó por Comunidades Autónomas (CCAA) de forma proporcional, en función del número de pacientes dependientes a opiáceos en cada una de las CCAA (Tabla I). 
Tabla I: Distribución de los investigadores y pacientes por Comunidades Autónomas

Andalucía: 200 pacientes (20 investigadores).

Asturias: 30 pacientes ( 3 investigadores).

Castilla-La Mancha: 30 pacientes (3 investigadores).

Canarias: 50 pacientes (5 investigadores).

Cataluña: 90 pacientes (9 investigadores).

Comunidad Valenciana: 70 pacientes ( 7 investigadores).

Extremadura: 20 pacientes ( 2 investigadores).

Galicia: 110 pacientes (11 investigadores).

La Rioja: 10 pacientes (1 investigador).

Madrid: 100 pacientes (10 investigadores).

Murcia: 40 pacientes (4 investigadores).

Navarra: 10 pacientes (1 investigador).

País Vasco: 40 pacientes (4 investigadores).

El número total previsto de investigadores/centros previstos fue de 80 y cada investigador/centro debía reclutar 10 pacientes a fin de cumplir con la cifra de 800 pacientes. Se incluyeron pacientes de ambos sexos que cumplían los criterios de ser mayores de 18 años, tener un diagnóstico de dependencia de opiáceos según los criterios diagnósticos del DSM-IV TR, estar en programas de terapia sustitutiva en centros de asistencia al paciente dependiente a opiáceos en el momento de la inclusión y firmar el consentimiento informado por escrito para participar en el estudio.

Además de los datos sociodemográficos, la caracterización clínica de las sustancias consumidas, la historia y pautas de tratamiento con el agonista opiáceo, se recogieron datos de la comorbilidad médica (infecciosas y no infecciosa) y psiquiátrica.

Para valorar la gravedad de la adicción de pacientes dependientes de opiáceos se utilizó el Addiction Severity Index (ASI) ampliamente utilizado en UE y USA ${ }^{13-14}$. El ASI es un instrumento válido que evalúa la gravedad de la adicción, frecuentemente utilizado en pacientes dependientes en heroína ${ }^{13}$ y en dependientes de opiáceos que están en tratamiento con metadona ${ }^{14}$.

Es un indicador de la gravedad clínica de los pacientes dependientes de heroína, al mismo tiempo que utilizado para diferenciar a los pacientes que han contactado con los 
dispositivos de salud mental de los que no ${ }^{13}$. También es útil como indicador del progreso en los $\mathrm{PMM}^{14}$. En pacientes en tratamiento con metadona unas puntuaciones bajas en el ASI y en la escala de incumplimiento predicen buena evolución ${ }^{15}$. Existe una versión Europea del ASI (EuropASI) ${ }^{16}$, en el que se han adaptado algunos ítems. Esta versión ha sido traducida y validada al castellano ${ }^{17}$.

\section{4-Conclusiones}

El estudio PROTEUS es un intento de conocer fielmente la realidad actual de los pacientes dependientes de opiáceos, atendidos en dispositivos de tratamiento. Se desarrolló en España entre septiembre del 2008 y Febrero del 2009.

Finalmente se incluyeron 624 pacientes procedentes de 74 centros repartidos por toda España. La edad media de los pacientes estudiados fue de 38,89 años siendo el $84 \%$ hombres.

Como objetivo principal permitirá describir el manejo del paciente dependiente de opiáceos atendido en los recursos asistenciales (tipo de tratamiento, dosis, evolución...).

Como objetivos secundarios permitirá describir los antecedentes personales, sociodemográficos, los problemas de conducción, estudiar la gravedad de la adicción, las comorbilidades médicas, infecciosas (tuberculosis, hepatitis por VHB y VHC, VIHSIDA) y no infecciosas y las psiquiátricas del eje I y II.

Se estudiarán los tratamientos agonistas empleados para el tratamiento de la dependencia de opiáceos y de sus posibles comorbilidades e interacciones tanto con psicofármacos, como los utilizados en el tratamiento de los problemas médicos.

\section{AGRADECIMIENTOS:}

El estudio PROTEUS se desarrolló gracias a la colaboración desinteresada del laboratorio Schering, especialmente de los miembros de su departamento médico: Belén Box y Jordi Llauradó.

En el estudio PROTEUS han participado: 
Addis Leonor de Álava Gelso , Aimee María Ruiz Rdriguez, Alfredo Gurrea Escajedo, Amhed Fabelo Laza, Ana Aparicio Aparicio, Ana $\mathrm{M}^{\mathrm{a}}$ Fernandez Sobrino, Ana Maria Germain Estebanez, Antonio Terán Prieto, Arancha López Mariano, Ariadna Balagué Añó, Bartolomé Baena San Juan, Bartolomé de la Fuente Darder, Begoña de Pablo García, Carlos Muralles Jiménez, Carlos Murga Cerviño, Carmen Beltran Porter, Carmen Cortell Cortell, Carmen Iglesias Azcue, Carmen Puerta García, Daniel Ángel Pereda Beaure, Desiderio Mejías Verdu, Francisco J. Samper Villar, Francisco Luque García, Garbiñe Caminos Valencia, Gemma Isabel San Narciso Izquierdo, Javier Ogando Rodríguez, Joaquín Martínez Valente, José A. González Aragón, José Antonio Segura Zamudio, Jose Luis Navarro González, José Manuel Fernández Fernández, José Martínez Raga, José Miguel Zoido Ramos, Juan Jesús Ruiz Ruiz, Juan Manuel Jiménez Lerma, Juan Ramírez López, Lucía Yolanda Armenteros García, Luis Garau Perello, Manuel Ruiz Martínez, Marco Antonio Rovira Isanda, Margarita Rossello del Rosal, María Elena Barbero García, María Olga Chapinal Sánchez, M ${ }^{a}$ Carmen Romero Truño, M Jesús Longo García Peñuela, Maria del Carmen García Nicolás, María del Mar Sánchez Fernández, María Jesús Antuña Díaz, María Lizaur Barbudo, María Paz Mateos Ayucar, Oscar Galera García, Pablo Vega Astudillo, Pedro A. de Armas Espinosa, Pedro Galindo Espada, Pilar Garzon Nacher, Rafael Forcada Chapa, Ricardo Ortega García, Ricardo Testa Garrido, Roberto Amador Curbelo, Roberto Fernando Artabe Noya, Tomás Díaz González, Tre Borras Cabaces, Víctor Puente Pazos, Visitación Villafuertes Marquez

\section{BIBLIOGRAFÍA}

1- Informe anual 2007: El problema de la drogodependencia en Europa. (2007) Observatorio. Europeo de las Drogas y las Toxicomanías. Recuperado el 10 de noviembre 2009 http://annualreport.emcdda.europa.eu

2-Plan Nacional sobre Drogas del Ministerio de Sanidad y Consumo http://www.pnsd.msc.es.

3- Álvarez FJ, González-Saíz F, Meana JJ, Pineda J, Roncero C. Documento de consenso de la sociedad española de toxicomanías sobre el empleo y la disponibilidad de la buprenorfina para el tratamiento de la dependencia de opiáceos en España. Trastornos adictivos 2005;7:44-6.

4- Roncero C., Sáez-Francàs N., Castells X.,Casas M. Eficacia y manejo clínico de la buprenorfina. Trastornos Adictivos 2008 10, (2): 77-87 
5-Alvarez FJ. El coste del tratamiento con buprenorfina en los pacientes dependientes de opiáceos. Gastar 4 millones de euros o dejar que mueran 8 pacientes. Conductas adictivas. $2004 ; 4: 1-2$

6-Terán A, Álvarez A, Sánchez R, Alvaro $\mathrm{M}^{\mathrm{a}}$ A. Complicaciones somáticas. diagnóstico y manejo de la infección por VIH, VHB, VHC y tuberculosis en el paciente heroinómano. Interacciones farmacológicas. Adicciones 2005; 17:123-65.

7- Roncero C, López-Ortiz C, Barral C, Sáez-Francàs N, Rovira M, Casas M. Tratamiento concomitante de litio y metadona en un paciente bipolar: a propósito de un caso. Adicciones. 2009; 21: 131-42

8-Rodríguez-Llera MC, Domingo-Salvany A, Brugal MT, Silva TC, Sánchez-Niubó A, Torrens M; ITINERE Investigators. Psychiatric comorbidity in young heroin users. Drug Alcohol Depend. 20061;84(1):48-55

9-Mateu G, Astals M, Torrens M. Comorbilidad psiquiátrica y trastorno por dependencia de opiáceos: del diagnóstico al tratamiento. Adicciones. 2005;17 Supl 2: $111-121$

10-Fernández Miranda J, González García-Portilla M, Sáiz Martínez P, Gutiérrez Cienfuegos E, Bobes García J. Influence of psychiatric disorders in the effectiveness of a long-term methadone maintenance treatment Actas Esp Psiquiatr. 2001;29(4):228-32

11-San Narciso G, Gutiérrez E, Sáiz P.A, González, M ${ }^{a}$ P, Bascarán, Mª T, Bobes, J. Evaluación de trastornos de la personalidad en pacientes heroinómanos mediante el International Personality Disorders Examination (IPDE). Adiciones 2000; 12 (1), 43-56

12-Torrens M, Serrano D, Astals M, Pérez-Domínguez G, Martín-Santos R Diagnosing comorbid psychiatric disorders in substance abusers: validity of the Spanish versions of the Psychiatric Research Interview for Substance and Mental Disorders and the Structured Clinical Interview for DSM-IV. Am J Psychiatry. 2004;161(7):1231-7. 13-Reelick NF, Wierdsma AI.The Addiction Severity Index as a predictor of the use of mental health care. Psychol Addict Behav. 2006; 20(2):214-8. 
14-Bovasso GB, Alterman AI, Cacciola JS, Cook TG. Predictive validity of the Addiction Severity Index's composite scores in the assessment of 2-year outcomes in a methadone maintenance population. Psychol Addict Behav. 2001; 15(3):171-6.

15-Craig RJ, Olson RE. Predicting methadone maintenance treatment outcomes using the Addiction Severity Index and the MMPI-2 Content Scales (Negative Treatment Indicators and Cynism scales). Am J Drug Alcohol Abuse. 2004; 30(4):823-39.

16-Kokeevei A, Hartgers C. European adaptation of a multidimensional assement instrument for drug and alcohol dependence. Eur Addict Res. 1995; 1 208-210

17-Bobes J, Bascarán MT, Bobes-Bascaán MT, Carballo JL, Díaz Mesa E, Flórez G, Garcia-Portilla MP, Saíz PA. Valoración de la Gravedad de la adicción: Aplicación a la Gestión clínica y monitorización de los tratamientos. Plan Nacional Sobre Drogas. Madrid. 2007 